\title{
Gestão de websites para eventos técnico-científicos suportados por um sistema de informação
}

\author{
Adriana G. Alves ${ }^{1}$, Andréia B. Bohner ${ }^{1}$ \\ ${ }^{1}$ Laboratório de Computação Aplicada - CTTMar - Universidade do Vale do Itajaí \\ (UNIVALI) \\ Caixa Postal 360 -88302-202 - Itajaí - SC - Brasil \\ \{adriana.alves, andreiabohner\}@univali.br
}

\begin{abstract}
This paper has as objective to present a tool that generates websites for technician-scientific events, supported by an information system for management of events. The tool allows a websites personalization, generating websites on-the-fly through programming techniques that use PHP, XML, CSS, Ajax and the data base Oracle. As a result we have an integrated solution to support events management, improving the creation of websites for events.
\end{abstract}

Resumo. Este artigo tem como objetivo apresentar uma ferramenta para geração de websites para eventos técnico-científicos, suportado por um sistema de informação para gestão de eventos. A ferramenta permite a personalização dos websites, gerando páginas on-the-fly através de técnicas de programação que utilizam PHP, XML, CSS, Ajax e o banco de dados Oracle. Como resultado tem-se uma solução integrada para o apoio a gestão de eventos, agilizando o procedimento de criação de websites para eventos.

\section{Introdução}

Com o crescente avanço das tecnologias e pesquisas nas mais diversas áreas científicas no Brasil, a necessidade de realização de eventos técnico-científicos para a troca de conhecimentos e divulgação de trabalhos vem se tornando cada vez mais freqüente nas instituições do país. Através destes eventos, pesquisadores apresentam os resultados de seus trabalhos e interagem com pessoas que estudam temas afins.

Nos bastidores destes eventos, muitas são as atividades relacionadas com todo o processo de organização, desde a divulgação do evento, submissão e avaliação de artigos à inscrição dos participantes, incluindo aí a geração de documentos para pagamento de taxas e todo controle financeiro. $O$ grande trâmite de informações e documentos faz necessária uma facilitação dos processos através da utilização de recursos computacionais que confiram à equipe organizadora do evento uma maior segurança e facilidade de acesso aos dados.

Uma das grandes necessidades da organização do evento é sua divulgação através de um site na Web para facilitar o acesso às informações, realização de inscrições e submissão de trabalhos. Para isso faz-se necessário o desenvolvimento da página Web por profissionais especializados e a hospedagem do website, o que implica em custos e dificuldades em se contratar serviços. Para os eventos que não são da área 
de tecnologia, há um agravante devido ao desconhecimento dos organizadores quanto à execução destes tipos de serviços.

Diante deste contexto foi desenvolvido um sistema de informação para dar suporte à gestão de eventos técnico-científicos através da Web, denominado Elis2 Sistema para Gerenciamento de Eventos Técnico-científicos (ELIS2, 2005). Este sistema, em funcionamento desde 2004, atende a diversas atividades dos eventos, no entanto não dá suporte para a geração de páginas dinâmicas dos eventos, as quais passaram a se tornar um gargalo para seus organizadores. Dessa forma, optou-se por criar uma ferramenta de fácil operação para geração de páginas Web dinâmicas para eventos, suportadas pelo Elis2.

Com a adoção do Elis2 e da ferramenta para geração de websites têm-se obtido agilidade nas tarefas relacionadas com a organização e divulgação de eventos, oferecendo um diferencial para a instituição organizadora e todos os envolvidos na realização de eventos técnico-científicos.

Este artigo tem como objetivo a apresentação da ferramenta de geração de websites para eventos, bem como as tecnologias adotadas para a mesma. $\mathrm{O}$ artigo está organizado da seguinte forma: no item 2 é apresentada a ferramenta para geração de websites de evento, e para uma melhor compreensão, é descrito brevemente o sistema de informação Elis2 que dá suporte à ferramenta. O item 2.1 descreve a funcionalidade da ferramenta apresentada, enquanto o item 2.2 apresenta as soluções computacionais adotadas. Por fim, no item 3, são apresentadas as limitações da ferramenta e as conclusões deste artigo.

\section{Ferramenta para geração de websites de eventos}

Para o organizador de um evento, é necessário publicar informações em um site específico e disponibilizar funções para submissão de artigos, inscrições e pagamentos via Web. A elaboração desses websites requer conhecimentos técnicos de computação e design os quais geralmente não são de domínio de usuários comuns desses serviços.

Existem para isso algumas ferramentas de autoria de páginas Web que são de fácil utilização, as quais oferecem recursos visuais e não requerem o conhecimento de linguagens, tais como HTML (Hyper Text Markup Language). Entretanto, no contexto da organização de eventos, normalmente tem-se a cada evento um grupo distinto de organizadores, os quais necessitariam de um treinamento para criarem suas próprias páginas $W e b$, ou a necessidade de contratação de serviços para este fim. Além da criação de páginas, é necessário o conhecimento sobre atividades de transferência de arquivos para um servidor Web, a criação de um domínio para o website, entre outras tarefas necessárias para a disponibilização desses serviços.

O Sistema de Informação Elis2 foi concebido para a gestão de eventos técnicocientíficos de modo a oferecer recursos para o apoio automatizado às tarefas envolvidas na organização das diversas fases e etapas de um evento. Originalmente desenvolvido para atender ao International Coastal Symposium (ICS2004, 2005), o sistema foi projetado para atender diversos eventos e conta hoje com mais de 50, tendo em sua base de dados aproximadamente 8500 pessoas, entre pesquisadores, autores, congressistas e 
palestrantes, provenientes de 42 países; 3200 trabalhos submetidos e 3650 inscrições realizadas.

O sistema Elis2 permite uma fácil adesão aos diversos modelos de eventos, através de configurações que possibilitam adequar suas funções às necessidades da organização. O sistema é orientado a $W e b$, o que facilita seu acesso em qualquer computador conectado à rede, bastando a utilização de um navegador que cumpra com as normas especificadas no HTML versão 3.2 do World Wide Web Consortium (W3C, 2004).

O Elis2 oferece dois ambientes distintos: (i) um módulo de administração restrito através do qual os organizadores configuram o evento e gerenciam as informações e (ii) um módulo público de serviços para submissão de artigos e inscrições nos eventos, fornecido através de links a serem inseridos no website do evento.

Desta forma, cabe ainda à organização do evento providenciar o desenvolvimento e hospedagem de uma página web para sua divulgação e utilizar os serviços públicos do Elis2 através dos links oferecidos. Com o uso crescente deste sistema de informação, a geração da página de divulgação dos eventos tornou-se um gargalo no fluxo de atividades desenvolvidas pela equipe, tornando necessário buscar-se uma solução que atendesse as necessidades dos diversos usuários para construção de suas páginas web.

Nesta perspectiva, optou-se por criar uma ferramenta de geração dos websites de eventos, a qual visa suprir as necessidades acima expostas, através de um ambiente de fácil utilização.

Para o desenvolvimento da ferramenta de geração de websites foi necessário um estudo de técnicas que oferecessem ao usuário um ambiente de fácil operação, não exigindo do mesmo profundos conhecimentos em qualquer tecnologia web. Assim, foi criada uma interface que se aproxima do padrão utilizado em ferramentas desktop e oferecidas opções que pudessem ser apresentadas de forma dinâmica através do banco de dados do sistema de administração do evento, Elis2.

\subsection{Funcionalidade da ferramenta de geração de websites}

A ferramenta de geração de websites para eventos técnico-científicos é acessada através do Sistema Elis2, após identificação do usuário na área restrita e seleção do evento desejado. A Figura 1 apresenta a tela (parcial) de entrada da ferramenta, onde se pode escolher o modelo de layout desejado para o website do evento.

O usuário pode optar por: (i) algumas opções extras de configuração que permitem a personalização do design do website (Figura 2 à esquerda), (ii) texto da homepage (Figura 2 à direita); (iii) fazer upload de imagens para o banner ou fundo da página (Figura 3 à esquerda), (iv) fontes (Figura 3 à direita), (v) selecionar cores de fundo, texto e navegadores (Figura 4); e por fim. (vi) definir as opções desejadas do menu (Figura 5). 


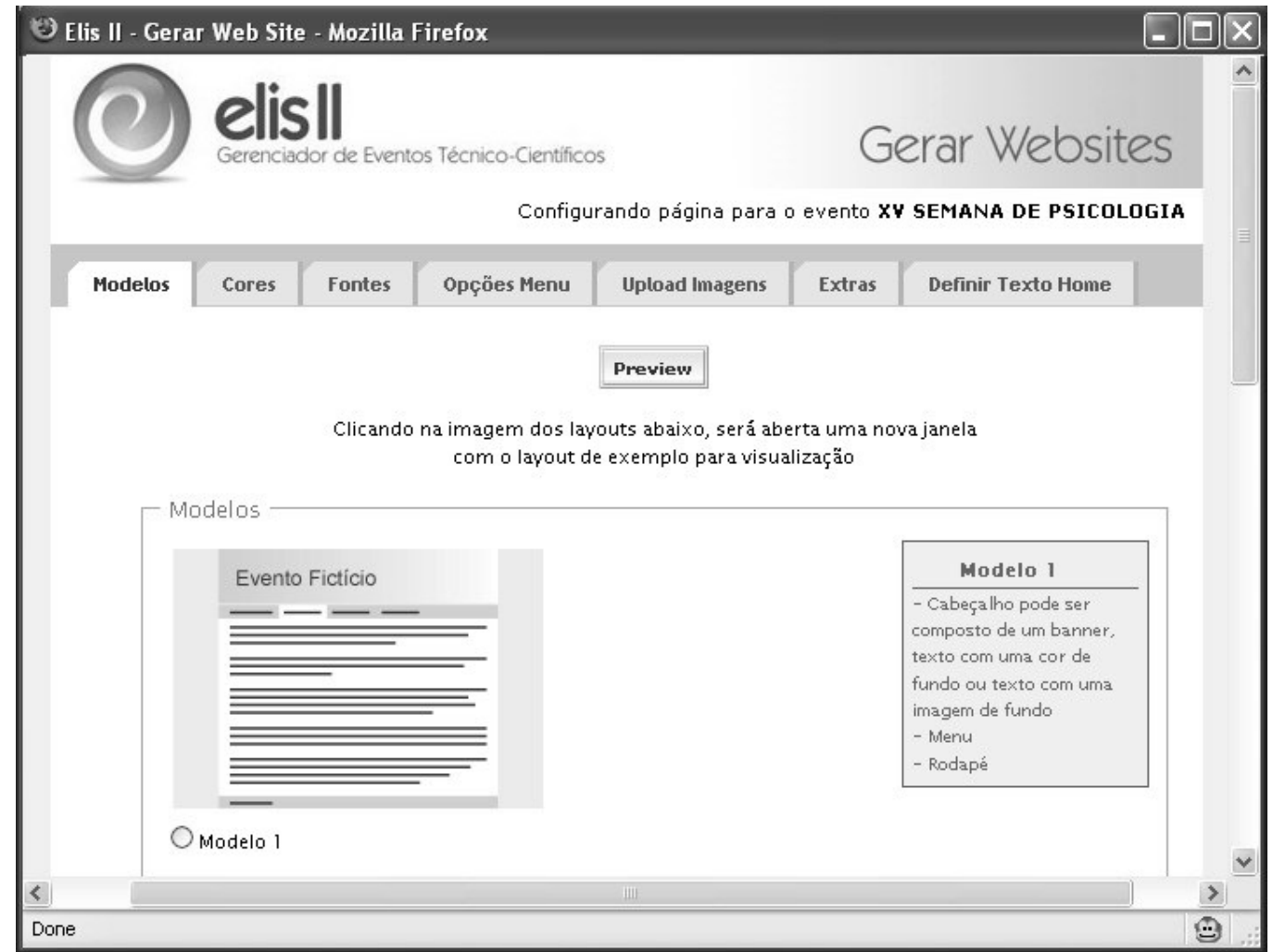

Figura 1 Ferramenta para Geração de Websites
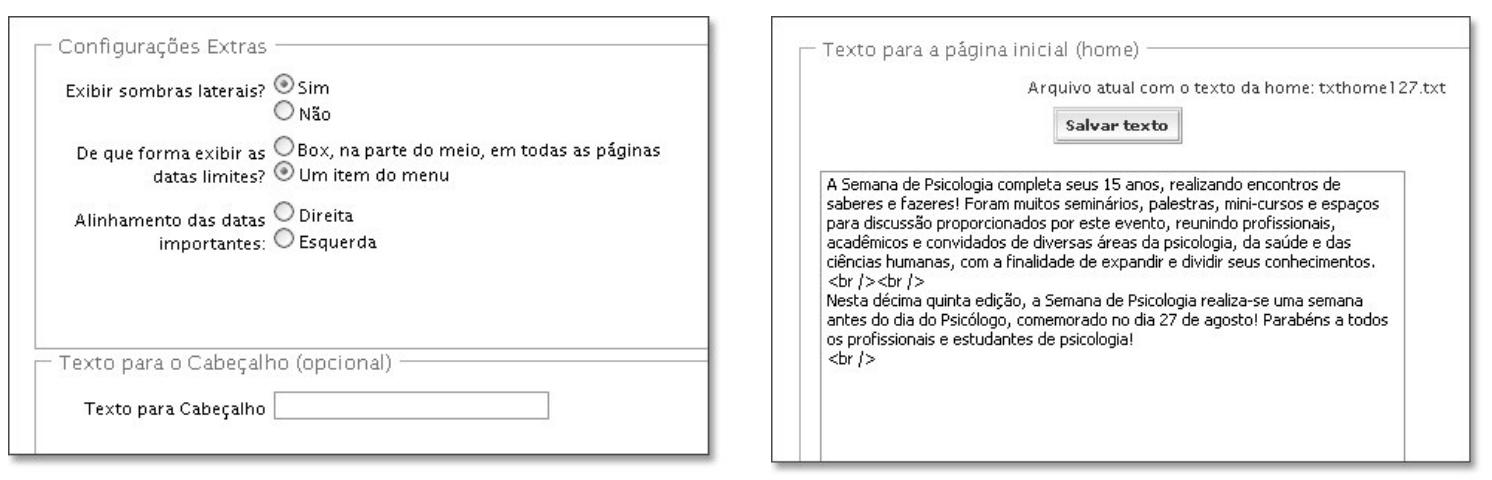

Figura 2 Configuração de extras e texto da home
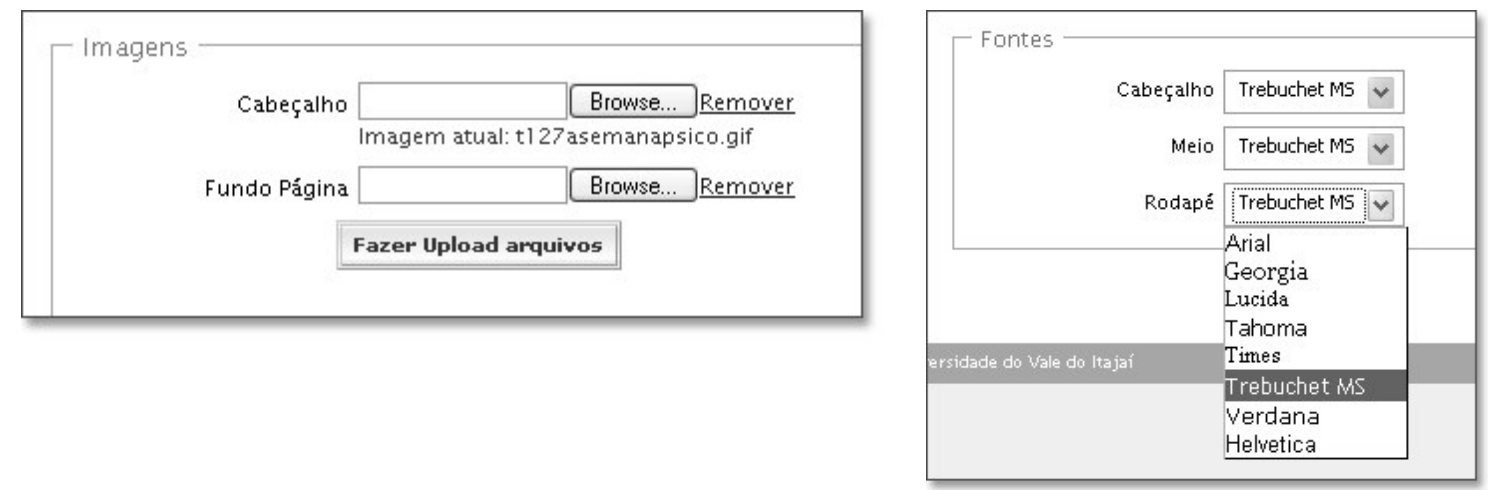

Figura 3 Importação de Imagens e Configuração de Fontes 


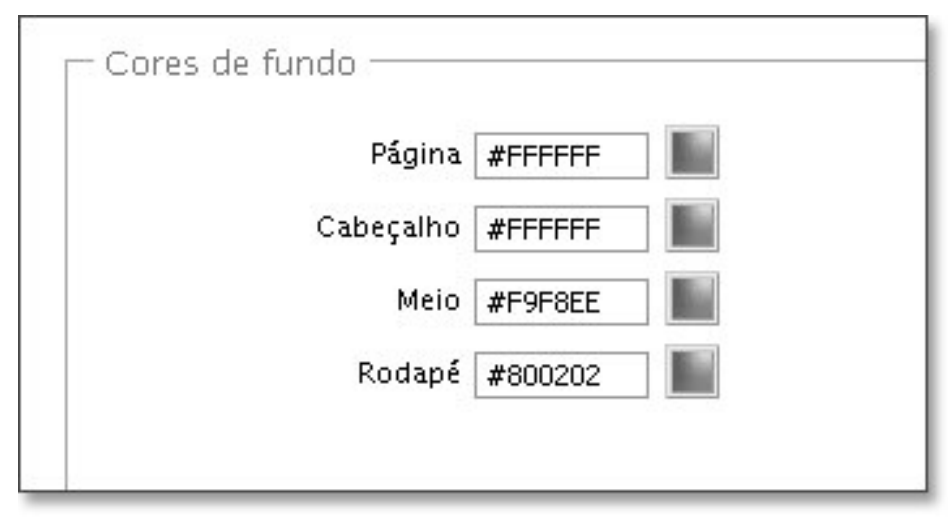

Figura 4 Configuração de cores da ferramenta

Ao item "opções de menu" dá-se um destaque, pois através do mesmo é realizado o acesso às funções do Elis2. Para cada item fornecido pela ferramenta e selecionado pelo organizador do evento, conforme Figura 5, serão apresentadas dinamicamente as informações previamente cadastradas no banco de dados do sistema de informação Elis2. No caso de inscrições e submissões, serão apresentadas as interfaces que permitem esta operação pelo usuário final, ou seja, autores de trabalhos e participantes do evento.

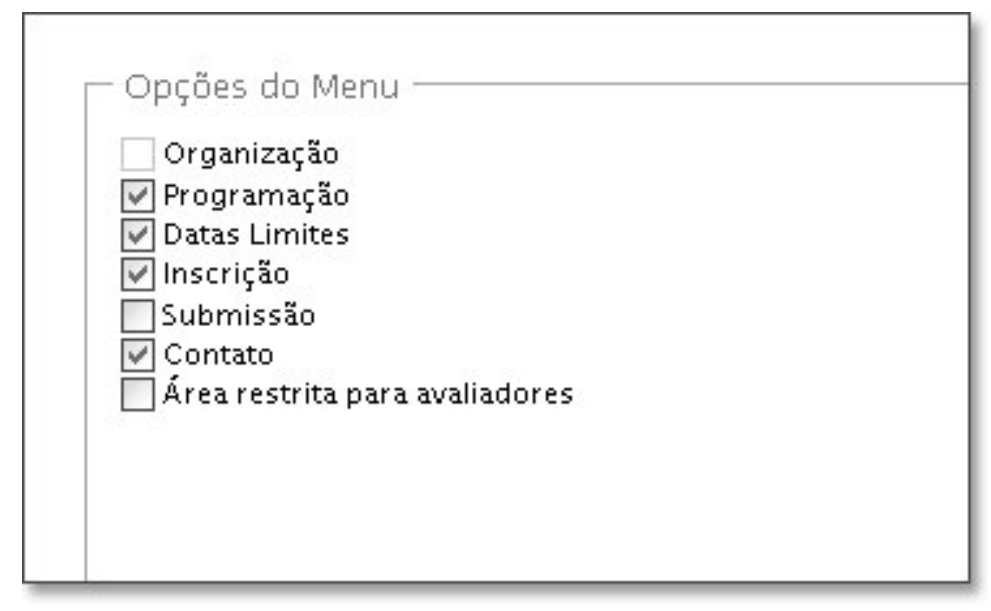

Figura 5 Opções de funcionalidade do Elis2

Ao finalizar as configurações, o usuário pode verificar como ficou a página através de um preview, e depois publicá-la, através da opção gerar site. Nesta última, não é realizada nenhuma operação de transferência de arquivos (FTP - File Transfer Protocol), pois as páginas são geradas dinamicamente, trazendo conteúdo do banco de dados e as configurações de um arquivo XML, conforme apresentado no item 2.2 deste artigo.

A publicação da página é feita automaticamente no website do Elis2, área pública (ELIS2, 2005), sem a necessidade de definição de um domínio próprio. Em casos de eventos que necessitem criar um domínio próprio na $\mathrm{W} e b$, basta criar um redirecionamento para o link disponibilizado pela ferramenta. A Figura 6 apresenta um exemplo de uma das páginas geradas pela ferramenta, no caso, a programação da XV 
Semana de Psicologia. Observa-se nesta figura um detalhamento da programação do evento, obtido através do banco de dados do Elis2.

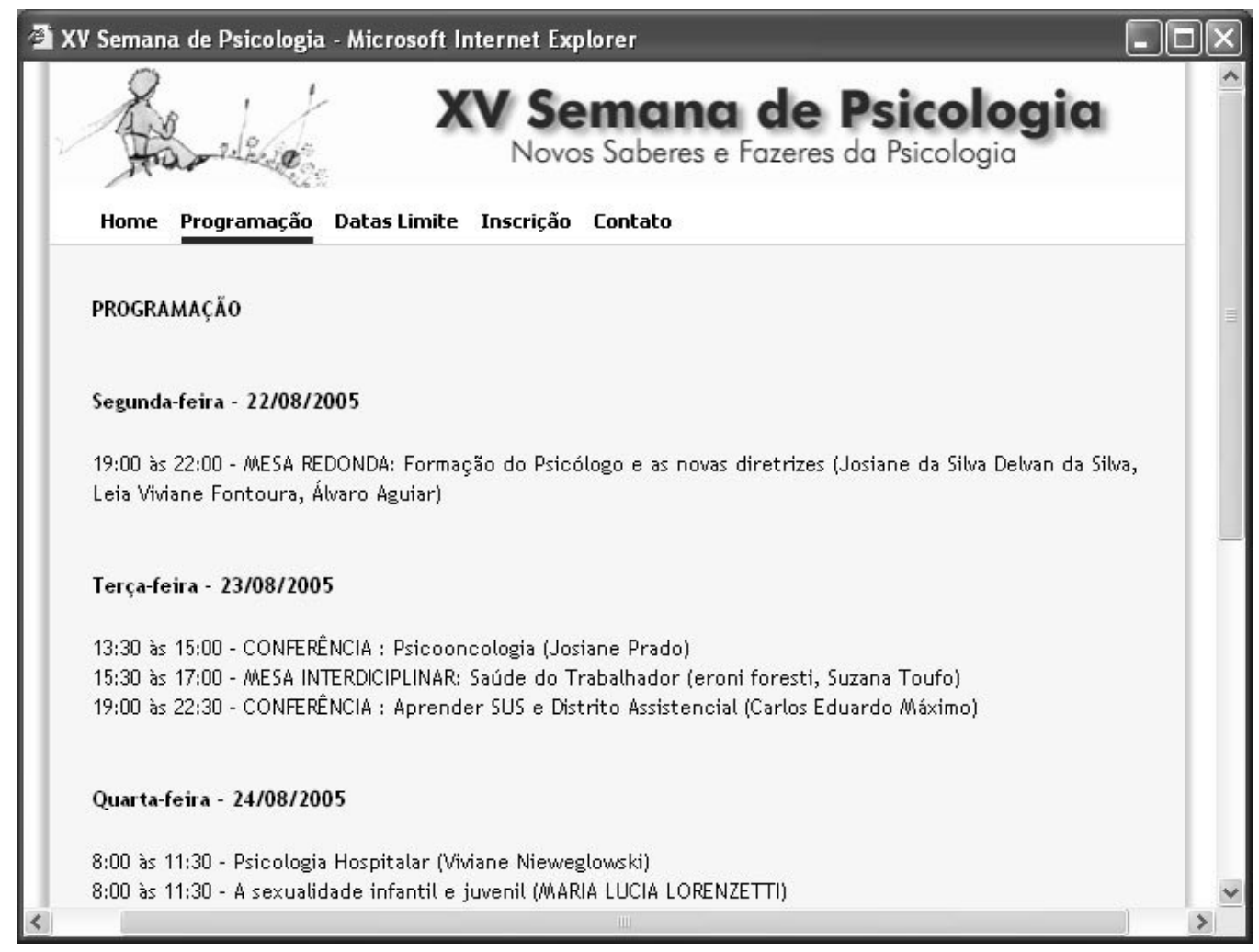

Figura 6 Exemplo de página gerada pela ferramenta

\subsection{Soluções Computacionais Adotadas}

Para o desenvolvimento da ferramenta de geração de websites dos eventos, foram utilizados o banco de dados Oracle (ORACLE, 2005), a linguagem de script PHP (Hypertext Preprocessor)(PHP, 2005), o esquema XML (Extensible Markup Language) (XML, 2005), modelos CSS e a técnica Ajax (Asynchronous JavaScript + XML) (MCLELLAN, 2005). O banco de dados Oracle foi utilizado devido ao fato que o Elis2 foi desenvolvido para esta ferramenta, no entanto é fácil utilizar outro SGBD desde que o mesmo utilize SQL Ansi.

A técnica Ajax (AJAX, 2005) permite realizar processamento no servidor (chamada de procedimento remoto), sem a necessidade de reload na página, o que melhora significantemente a usabilidade das páginas, tornando a interface com o usuário mais fácil de trabalhar, mais rápida e aproximando a semelhança com as interfaces dos aplicativos desktop, com os quais os usuários já estão mais familiarizados.

O esquema XML foi utilizado para gravar as configurações que os usuários definem para os websites através da ferramenta. Conforme as configurações das páginas são selecionadas, o arquivo XML é gerado dinamicamente para armazenar as mesmas, sendo gerado um arquivo de configuração XML para cada evento.

Os layouts dos modelos de websites disponíveis na ferramenta são definidos através de um arquivo CSS (Cascading Style Sheet), que também é gerado 
dinamicamente através de um script PHP, responsável por gerar o CSS conforme o modelo selecionado, com as configurações obtidas do arquivo XML.

Os websites dos eventos são gerados através de uma página PHP, que é acessada passando um único parâmetro - o código identificador do evento - e, a partir desta informação, todas as páginas do evento são geradas on-the-fly, sendo as configurações do website trazidas do arquivo XML e, aplicadas ao arquivo CSS, conforme o modelo selecionado. O conteúdo das páginas é fornecido através do banco de dados Oracle, que é alimentado através da parte administrativa do sistema Elis2. A Figura 7 apresenta de forma esquemática a geração das páginas do website dos eventos.

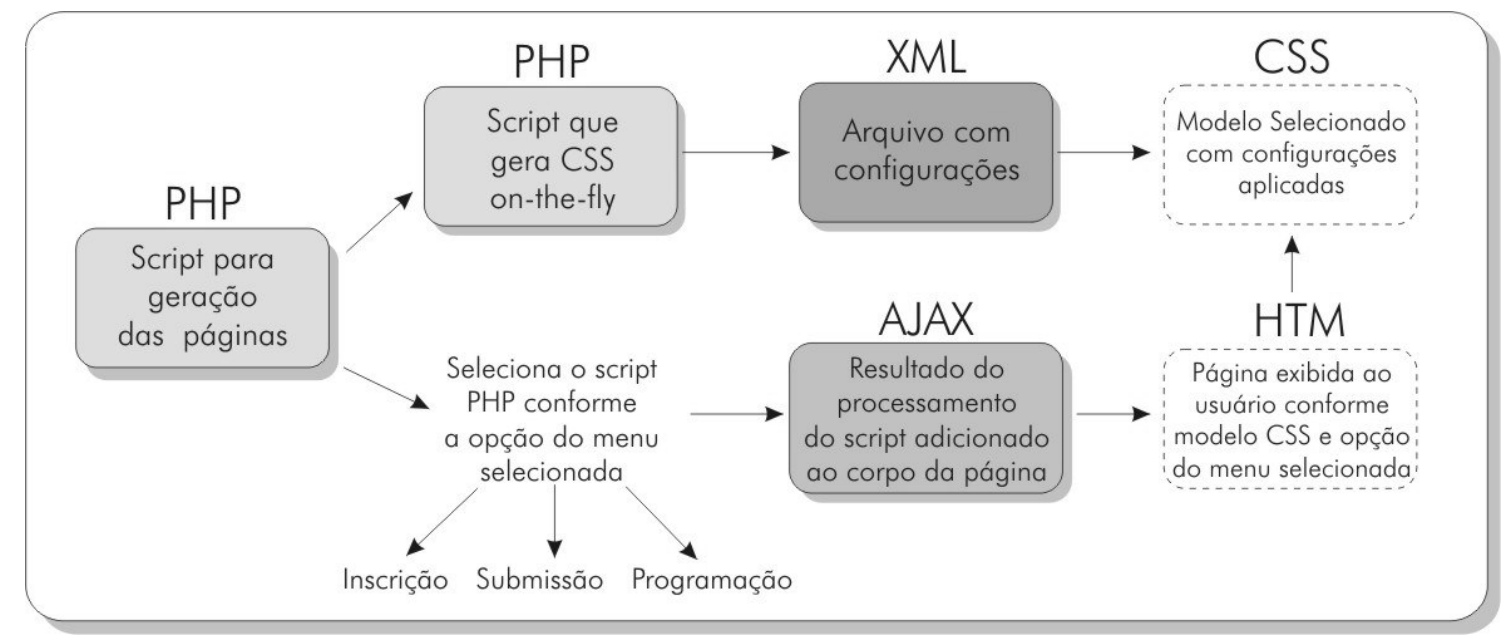

Figura 7 Esquema de Geração on-the-fly das Páginas do Website do Evento

\section{Conclusões}

A implantação de uma ferramenta para geração de websites para eventos técnicocientíficos veio ao encontro da necessidade de organizadores de eventos em divulgar informações e gerenciar submissões e inscrições dos eventos que organizam. Esta atividade vinha se mostrando um gargalo na gestão de eventos, pois, mesmo que o Sistema de Informações Elis2 suprisse funções fundamentais, as mesmas não podiam ser disponibilizadas de forma ágil e simples para os usuários.

O uso de técnicas que permitem a geração dinâmica de páginas Web sem a necessidade de conhecimentos profundos em computação constitui-se num diferencial para um sistema de informação e amplia a possibilidade do uso das informações contidas no seu banco de dados.

Buscou-se neste trabalho aplicar novas técnicas de programação, como o Ajax, para criar páginas com melhor usabilidade, item fundamental para uma fácil aceitação do usuário à ferramenta. Também o XML permitiu grande agilidade para $\mathrm{o}$ armazenamento e recuperação da configuração construída pelo usuário, tornando a geração das páginas totalmente on-the-fly, quando somada ao acesso ao banco de dados do sistema de informação para gestão de eventos.

A ferramenta para geração de websites limita-se a modelos pré-definidos de websites de eventos, o que pode não atender a diferentes estruturas de layouts, por vezes desejáveis em páginas de eventos. Nestes casos, o usuário deverá desenvolver sua 
própria página e utilizar o Elis2 através de links para as funções de inscrição e submissão de artigos.

Por fim, a ferramenta descrita neste artigo prescinde de um sistema de informação robusto e maduro, o Elis2, e está permitindo uma padronização da geração de websites para a instituição que a utiliza, facilitando e agilizando os procedimentos, tendo como ponto forte a personalização necessária e desejada a cada novo evento que utiliza seus serviços.

\section{Referências}

AJAX. Ajax: A New Approach to Web Applications. Disponível em: http://www.adaptivepath.com/publications/essays/archives/000385.php. Acesso em: 01 julho 2005.

ELIS2. Sistema para Gerenciamento de Evento Técnico-Científicos. Laboratório de Computação Aplicada - CTTMar - Univali, 2005. Disponível em: $<$ www.univali.br/eventos $>$.

ICS2004. 8th International Coastal Symposium. March 14-19, 2004, Santa Catarina, Brazil. Coordenador Antonio Henrique da Fontoura Klein. Disponível em: ICS2004 http://siaiacad05.univali.br/ ics2004/. Acesso em: 15 de agosto de 2005.

MCLELLAN, Drew. Very Dynamic Web Interfaces. 2005. Disponível em: http://www.xml.com/pub/a/2005/02/09/xml-http-request.html. Acesso em: 01 julho 2005.

ORACLE. Página oficial do SGBD Oracle. Disponível em: http://www.oracle.com/. Acesso em: 03 julho 2005.

PHP. Página oficial da PHP - Hypertext Preprocessor. Disponível em: http://www.php.net/ Acesso em: 5 julho 2005.

W3C. Apresenta dados referentes ao World Wide Web Consortium. Disponível em: http://www.w3c.org/ Acesso em: 2 abril 2004.

XML. Página contendo informações e soluções para XML - Extensible Markup Language.Disponível em http://www.xml.com/. Acesso em: 2 julho 2005. 